\title{
ROSENBROCK-TYPE METHODS ADAPTED TO DIFFERENTIAL-ALGEBRAIC SYSTEMS
}

\author{
CLAUS SCHNEIDER
}

\begin{abstract}
We consider the numerical solution of differential-algebraic systems of index one given in Kronecker canonical form. The methods described here are derived from the Rosenbrock approach. Hence, they do not require the solution of nonlinear systems of equations but one evaluation of the Jacobian and one LU decomposition per step. By construction, the $s$-stage method coincides with a solver for nonlinear equations of order $s+1$ if the stepsize is set to zero. In this sense, the adaptation to differential-algebraic equations is performed. The special structure of the method leads to simplified order conditions and to an easy implementation. Some particular methods up to order 4 are given. Especially, an embedded 4-stage method of order $4(3)$ is derived.
\end{abstract}

\section{INTRODUCTION}

In this paper we will be concerned with differential-algebraic equations (DAE's) of the Kronecker canonical form

$$
\begin{array}{cc}
y^{\prime}=f(y, z), & 0=g(y, z), \\
y\left(x_{0}\right)=y_{0}, & z\left(x_{0}\right)=z_{0},
\end{array}
$$

where the initial values (1.2) are assumed to be consistent, i.e., $g\left(y_{0}, z_{0}\right)=0$. Furthermore, we assume that $f$ and $g$ are sufficiently smooth functions and that $\partial g / \partial z$ has a bounded inverse in a neighborhood of the exact solution, i.e., the DAE is of index one. Then local existence, uniqueness, and regularity of the solution are assured (cf. [3, 10,13]). Obviously, the case of an implicitly given ordinary differential equation $F\left(y, y^{\prime}\right)=0$ is included in (1.1) with $f(y, z):=$ $z$ and $g:=F$. For the numerical solution we will study a Rosenbrock-type method (ROW method), i.e., a linearly implicit Runge-Kutta method applied to (1.1). But the ROW method will be modified in such a way that it contains a high-order solver for purely nonlinear equations. Other approaches have been published in $[1,2,4,6-8,11]$.

The paper is organized as follows. In $\S 2$ we describe the ROW methods and their applications to DAE's. In $\S 3$ we discuss the order of such a method in the

Received August 7, 1989.

1980 Mathematics Subject (lassification (1985 Revision). Primary 65L05, 58F99.

Key words and phrases. Differential-algebraic equations, Rosenbrock type methods, implicitly given differential equations, implicit function, homotopy-methods. 
context of DAE's, also for especially simple DAE's with $f=1$ and $y\left(x_{0}\right)=x_{0}$ (i.e., for the problem of following the implicitly defined function $z$ ), and for the solution of nonlinear equations. This leads (in $\S 4$ ) to a class of ROW methods adapted to DAE's. Finally, in $\S 5$, some particular methods of order 2, 3, and 4 are given.

\section{ROW METHODS}

ROW methods are linearly implicit Runge-Kutta methods and may be considered as modified Rosenbrock methods. They were introduced by Wolfbrandt in [16] and extensively studied by Nørsett and Wolfbrandt in [9] and by Kaps and Wanner in [5], who also described a transformed version which is more suitable for numerical computations. ROW methods are easy to derive from singly diagonally implicit Runge-Kutta methods (SDIRK): Let $A$ be a lower triangular matrix with $\operatorname{diag}(A)=\gamma I, \gamma \neq 0$. Then the first step of an $s$-stage SDIRK for the solution of the initial value problem

$$
y^{\prime}=f(y), \quad y\left(x_{0}\right)=y_{0}
$$

is given by

$$
\begin{gathered}
k_{i}=h f\left(y_{0}+\sum_{j=1}^{i} a_{i j} k_{j}\right), \quad i=1(1) s, \\
y_{1}=y_{0}+\sum_{i=1}^{s} \mu_{i} k_{i} .
\end{gathered}
$$

Now linearize (2.2) with respect to $\Gamma$, where $A=\alpha+\Gamma, \alpha$ and $\Gamma$ lower triangular matrices, $\operatorname{diag}(\alpha)=0, \operatorname{diag}(\Gamma)=\gamma I$. This process yields the ROW method

$$
k_{i}=h f\left(y_{0}+\sum_{j=1}^{i-1} \alpha_{i j} k_{j}\right)+h f_{y}^{0} \sum_{j=1}^{i} \gamma_{i j} k_{j}, \quad i=1(1) s,
$$

where $f_{y}^{0}$ denotes the Jacobian of $f$ at $y_{0} . \Gamma=\operatorname{diag}(A)$ leads to the classical Rosenbrock process (cf. [15]). Since $\Gamma$ is regular, the transformed version of Kaps and Wanner is achieved by setting $\kappa_{i}:=\sum_{j=1}^{i} \gamma_{i j} k_{j}, i=1(1) s$ :

$$
\begin{aligned}
\left(I-h \gamma f_{y}^{0}\right) \kappa_{i}= & \gamma h f\left(y_{0}+\sum_{j=1}^{i-1}\left(\alpha \Gamma^{-1}\right)_{i j} \kappa_{j}\right) \\
& -\gamma \sum_{j=1}^{i-1}\left(\Gamma^{-1}\right)_{i j} \kappa_{j}, \quad i=1(1) s,
\end{aligned}
$$

$$
y_{1}=y_{0}+\sum_{i=1}^{s}\left(\mu^{\mathrm{T}} \Gamma^{-1}\right)_{i} \kappa_{i}
$$


Hence, the matrix-vector product in (2.4) is avoided. Only one LU decomposition of $\left(I-h \gamma f_{y}^{0}\right)$ per step is required, independently of $s$ - the number of stages. The ROW method is defined by $A=\alpha+\Gamma$ and $\mu$. Now Roche applied this method to the DAE given in (1.1). Furthermore, he derived order conditions and computed the coefficients of some numerical methods in his excellent paper [14]. Then (2.4), (2.3) read as follows:

$$
\begin{gathered}
a_{i}:=y_{0}+\sum_{j=1}^{i-1} \alpha_{i j} k_{j}, \quad b_{i}:=z_{0}+\sum_{j=1}^{i-1} \alpha_{i j} l_{j}, \quad i=1(1) s, \\
k_{i}=h f\left(a_{i}, b_{i}\right)+h f_{y}^{0} \sum_{j=1}^{i} \gamma_{i j} k_{j}+h f_{z}^{0} \sum_{j=1}^{i} \gamma_{i j} l_{j}, \quad i=1(1) s, \\
0=g\left(a_{i}, b_{i}\right)+g_{y}^{0} \sum_{j=1}^{i} \gamma_{i j} k_{j}+g_{z}^{0} \sum_{j=1}^{i} \gamma_{i j} l_{j}, \quad i=1(1) s, \\
y_{1}=y_{0}+\sum_{i=1}^{s} \mu_{i} k_{i}, \quad z_{1}=z_{0}+\sum_{i=1}^{s} \mu_{i} l_{i} .
\end{gathered}
$$

Now the matrix

$$
\left[\begin{array}{cc}
I-h \gamma f_{y}^{0} & -h \gamma f_{z}^{0} \\
g_{y}^{0} & g_{z}^{0}
\end{array}\right]
$$

has to be decomposed in every step. Obviously, a transformed version may be deduced in this case too. But it is not at all obvious why the same matrix $\Gamma$ is used in (2.8) and in (2.9), which are linearizations of two basically different types of equations. This choice has some practical benefits, as will be seen later, but it is not compelling. Therefore, we will replace $(2.9)$ by

$$
0=g\left(a_{i}, b_{i}\right)+g_{y}^{0} \sum_{j=1}^{i} \delta_{i j} k_{j}+g_{z}^{0} \sum_{j=1}^{i} \delta_{i j} l_{j}, \quad i=1(1) s,
$$

with $B:=\alpha+\Delta, \Delta$ a regular lower triangular matrix. Furthermore, we no longer assume $\gamma_{i i}=\gamma$ for $i=1(1) s$. Then the matrix to be decomposed becomes

$$
\left[\begin{array}{cc}
I-h \gamma_{i i} f_{y}^{0} & -h \gamma_{i i} f_{z}^{0} \\
g_{y}^{0} & g_{z}^{0}
\end{array}\right]
$$

at stage $i$, showing that it makes sense to require only $\gamma_{i i} \in\{\gamma, 0\}, \gamma \neq 0$, $i=1(1) s$. If $\operatorname{diag}(\Gamma)=0$, then (2.8) is an explicit equation. The same is true in a method with $\Gamma=0$ studied by Rentrop, Roche, and Steinebach in [12]. There, $\alpha, \mu$ are taken from an explicit Runge-Kutta-Fehlberg method, and $\Delta$ is used to fulfill the remaining order conditions. The coefficients of a 6-stage method of order 4 are also given in [12]. We intend to use the matrix $\Delta$ as a tool for the efficient solution of nonlinear equations-(2.7)-(2.10) reduces to such a problem if $h=0$. Then $\alpha, \Gamma$, and $\mu$ are the remaining parameters (just as in Roche's approach), which have to be chosen such that a high-order 
method is achieved. The order equations for the methods (2.7)-(2.10) and (2.7), (2.8), (2.11), (2.10) are summarized in Table 1, where the following conventions are used: $e$ denotes the vector $(1,1, \ldots, 1)^{\mathrm{T}} \in \mathbb{R}^{s} ;$ if $y, x \in \mathbb{R}^{s}$ and $\nu \in \mathbb{N}$, then $x^{\nu}$ denotes the vector $\left(x_{1}^{\nu}, x_{2}^{\nu}, \ldots, x_{s}^{\nu}\right)^{\mathrm{T}}$, and $x \cdot y$ the vector $\left(x_{1} y_{1}, \ldots, x_{s} y_{s}\right)^{\mathrm{T}}$.

TABLE 1

Order conditions for order $\rho$

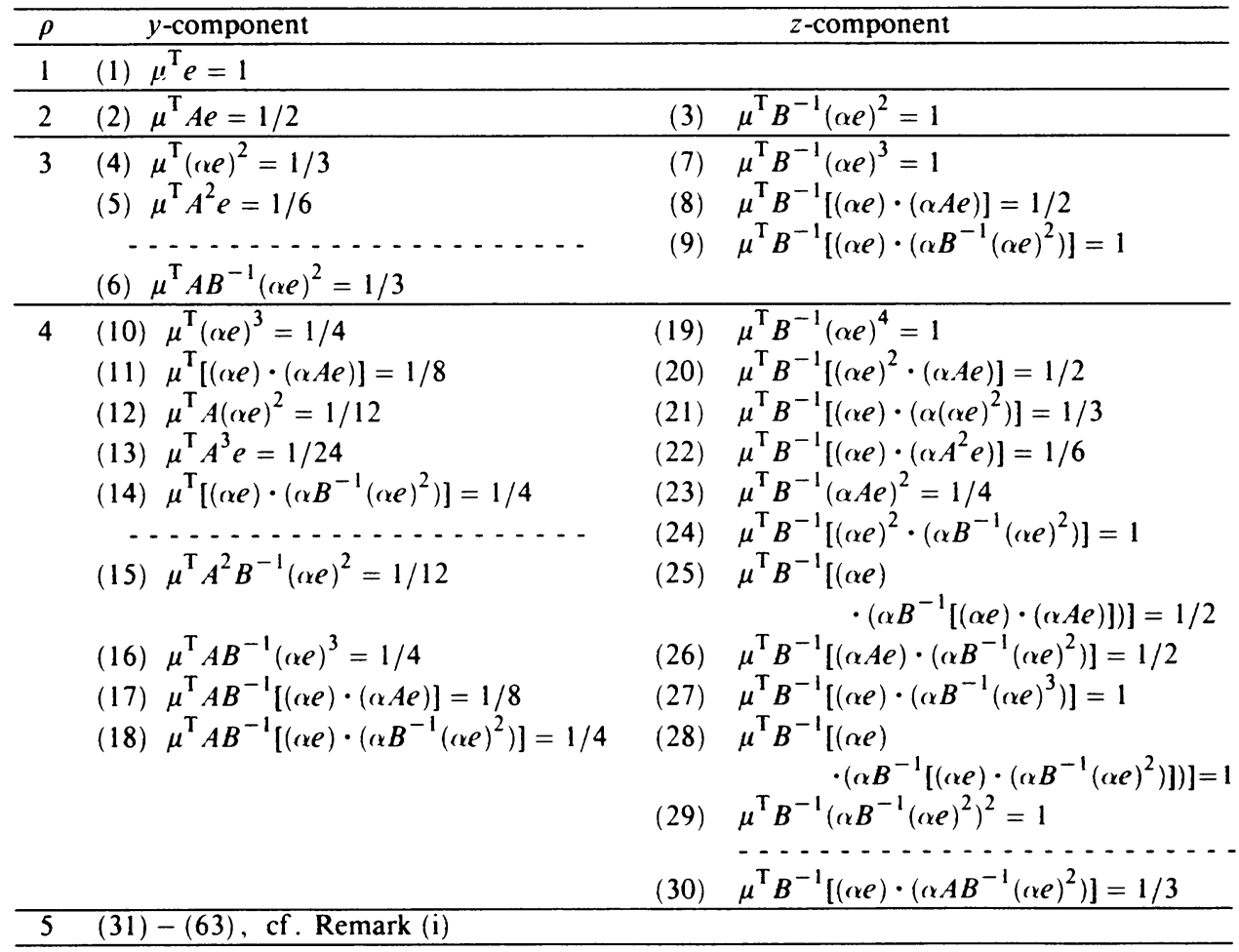

Remarks. (i) The order conditions (1)-(5) and (7)-(14) are given in [14], and (6), (15)-(18) in [12]. The remaining conditions (19)-(63) have been computed by W. Link (University of Mainz, private communication) according to the rules of [14]. His results show that the not explicitly given conditions (31)-(63) may be deduced from the conditions $(10)-(30)$ in the following manner:

(a) If $\mu^{\mathrm{T}} x=\sigma$ is condition $(i), i \in\{10, \ldots, 30\}$, then condition $(21+i)$ has the form $\mu^{\mathrm{T}} A x=\sigma / 5$.

(b) If $\mu^{\mathrm{T}} B^{-1} x=\sigma$ is condition $(i), i \in\{19, \ldots, 30\}$, then condition $(33+i)$ has the form $\mu^{\mathrm{T}} x=\sigma / 5$.

(ii) The order equations without $B^{-1}$ describe just the order conditions for the ODE case given in [5]. 
(iii) If $\Delta=\Gamma$, then the equations containing $A \cdot B^{-1}-(6),(15)-(18)$, and (30)-are redundant, e.g., (30) is already fulfilled by virtue of (21). Hence, $\Delta \neq \Gamma$ introduces six additional equations in Table 1 , and 17 for $\rho=5$ in the $y$-component.

\section{ORDER OF THE METHOD}

The following theorem connects the order conditions and a requirement for stability, yielding a result on convergence of the ROW method.

Theorem (Convergence). Consider the method (2.7), (2.8), (2.11), (2.10), and assume the following.

(i) The order conditions of the $y$-component hold for $\rho=1(1) p$.

(ii) The order conditions of the $z$-component hold for $\rho=2(1) p-1$.

(iii) The stability condition

$$
-1 \leq 1-\mu^{\mathrm{T}} B^{-1} e<1
$$

holds.

Then the ROW method is convergent of order $p$, i.e., for $n \cdot h$ fixed,

$$
\max _{0 \leq j \leq n}\left|y_{j}-y\left(x_{0}+j h\right)\right|=O\left(h^{p}\right)
$$

and

$$
\max _{0 \leq j \leq n}\left|z_{j}-z\left(x_{0}+j h\right)\right|=O\left(h^{p}\right) .
$$

Proof. The proof follows by collecting results from $[1,4,14]$.

Applied to an ordinary differential equation, i.e., $f_{z}=0, g=0$ in (1.1), a $p$ th order ROW method obviously converges with order $p$ at least. If, on the other hand, the DAE to be solved has the form

$$
y^{\prime}=1, \quad g(y, z)=0, \quad y\left(x_{0}\right)=x_{0}, \quad g\left(y_{0}, z_{0}\right)=0,
$$

i.e., $f_{z}=0, f_{y}=0$, then we are dealing with the problem of computing the implicitly defined function $z$ at points $x_{0}+n h$, knowing its value at $x_{0}$. Thus, applying the ROW method to a problem with a special structure as in (3.2) yields an efficient algorithm for following the path of an implicitly given function. Furthermore, high-order ROW methods designed for this special problem may be derived by cancelling those order conditions in Table 1 which hold independently of the coefficients $\alpha, \Gamma, \Delta, \mu$ if $f_{y}=0$ and $f_{z}=0$. From Roche's results in [14] it is easy to deduce the following lemma.

Lemma. Consider the ROW method (transformed version) applied to (3.2):

$$
\lambda_{i}=-\left(g_{z}^{0}\right)^{-1}\left[g\left(x_{0}+h(\alpha e)_{i}, z_{0}+\sum_{j=1}^{i-1}\left(\alpha \Delta^{-1}\right)_{i j} \lambda_{j}\right)+h g_{y}^{0}(\Delta e)_{i}\right]{ }_{i=1(1) s}
$$

$$
z_{1}=z_{0}+\sum_{i=1}^{s}\left(\mu^{\mathrm{T}} \Delta^{-1}\right)_{i} \lambda_{i}
$$


Assume that

(i) $\mu^{\mathrm{T}} e=1$ (preconsistency).

(ii) $-1 \leq 1-\mu^{\mathrm{T}} B^{-1} e<1$ (stability).

(iii) The order conditions for the z-component with right-hand side equal to one hold for $\rho=2(1) p-1$.

Then the ROW method (3.3), (3.4) is convergent of order $p$, i.e., for $n h$ fixed, $y_{j}=x_{0}+j h, 0 \leq j \leq n$, and $\max _{0 \leq j \leq n}\left|z_{j}-z\left(x_{0}+j h\right)\right|=O\left(h^{p}\right)$.

In order to achieve $p=5$, e.g., the equations to be satisfied are (1), (3), (7), (9), (19), (24), (27)-(29) in Table 1, and the stability condition (3.1).

Example of an ROW method for the solution of (3.2). Let $R:=\left(r_{i j}\right)_{i, j=1(1) s}$ with $r_{i j}=1, i \geq j, r_{i j}=0$ otherwise, and $e_{j}$ be the $j$ th unit vector in $\mathbb{R}^{s}$. With $B:=\operatorname{diag}(1 / i) R, \alpha:=\left(I-R^{-1}\right) B, \Delta=B-\alpha=R^{-1} B$, and $\mu:=e / s$, we have $\alpha e=e-e_{1},\left(\alpha \Delta^{-1}\right)_{i j}=1$ for $i>j, \Delta e=e_{1}$, and $\mu^{\mathrm{T}} \Delta^{-1}=e^{\mathrm{T}} B^{-1} R / s=e_{s}^{\mathrm{T}} R=e^{\mathrm{T}}$. Then (3.3), (3.4) become

$$
\lambda_{1}=-\left(g_{z}^{0}\right)^{-1}\left[g\left(x_{0}, z_{0}\right)+h g_{y}^{0}\right] \text {, }
$$

$$
\lambda_{i}=-\left(g_{z}^{0}\right)^{-1} g\left(x_{0}+h, z_{0}+\sum_{j=1}^{i-1} \lambda_{j}\right), \quad i=2(1) s,
$$

$$
z_{1}=z_{0}+\sum_{i=1}^{s} \lambda_{i}
$$

This special $s$-stage ROW method is of order $s+1$ for problem (3.2), as can be seen very easily, at least for $s \leq 4$, from the order equations, since

(3.7) $\quad \mu^{\mathrm{T}} B^{-1}=e_{s}^{\mathrm{T}}$ and $\alpha B^{-1}=I-R^{-1}=$ shift to the right .

Hence, stability and preconsistency are assured by the ansatz. The remaining order conditions simplify to

$$
(\alpha e)_{2}^{2}=1, \quad(\alpha e)_{j}=1 \text { for } j=3(1) s,
$$

and they are obviously satisfied. That the $s$-stage method (3.5), (3.6) is of order $s+1$ for arbitrary $s$ can be seen by inspecting the order trees for $(3.3),(3.4)$ and the theorems given by Roche in [14]. In this special case the remaining $z$-trees of order $\rho$ are constructed recursively starting with the order-two $z$-tree:

Let $t$ be a $z$-tree of order $\rho-1 \geq 2:$ (a) add an edge with a meager vertex to a fat vertex of $t$ or (b) replace a meager vertex of $t$ by the order-two $z$-tree.

The resulting trees are the $z$-trees of order $\rho$ for method (3.3), (3.4) applied to problem (3.2). Employing these rules in the order equations and using the special structure of the method (3.5), (3.6), i.e., using (3.7), yields the conditions (3.8).

Remark. If, in (3.2), $x_{0}=0$ and $g(y ; z)=\varphi(z)-(1-y) \varphi\left(z_{0}\right)$, then (3.4), (3.5) defines a homotopy method for finding a root $z=z(1)$ of $\varphi$. It works as long as $g_{z}=\varphi_{z}$ is regular and the DAE possesses a solution from 0 to 1 . 
After this study of some rather special DAE's we now inspect the case $h=$ 0 in the general algorithm. Then $k_{i}=0, i=1(1) s$, and the $l_{i}$ are given recursively. In the transformed version (cf. (3.3)) we have

$$
\lambda_{i}=-\left(g_{z}^{0}\right)^{-1} g\left(y_{0}, z_{0}+\sum_{j=1}^{i-1}\left(\alpha \Delta^{-1}\right)_{i j} \lambda_{j}\right), \quad i=1(1) s
$$

and the next iterate is given by

$$
z_{1}=z_{0}+\sum_{i=1}^{s}\left(\mu^{\mathrm{T}} \Delta^{-1}\right)_{i} \lambda_{i}
$$

If $s=1$, then (3.10) describes one step of a damped Newton method with damping factor $\mu_{1} / \delta_{11}=1 / \delta_{11}$ for a consistent ROW method. Hence, only $\delta_{11}=1$ yields a quadratically convergent method for the solution of $g\left(y_{0}, z\right)=$ 0 . If $s>1$, then a method of order $s+1$ (order means $Q$-order of the solver (3.9), (3.10) for nonlinear equations) is achieved if

$$
\left(\alpha \Delta^{-1}\right)_{i j}=1, i>j, \quad \text { and } \mu^{\mathrm{T}} \Delta^{-1}=e^{\mathrm{T}} .
$$

Indeed, this choice leads to an iterative method of order $s+1$ which was introduced and discussed by Traub in [17, pp. $165 \mathrm{ff}$ and pp. $174 \mathrm{ff}]$ :

$$
\begin{gathered}
\lambda_{i}=\left(-g_{z}^{0}\right)^{-1} g\left(y_{0}, z_{0}+\sum_{j=1}^{i-1} \lambda_{j}\right), \quad i=1(1) s \\
z_{1}=z_{0}+\sum_{i=1}^{s} \lambda_{i} .
\end{gathered}
$$

In the following section we construct ROW methods with property (3.11). Hence, they coincide with Traub's algorithm if $h=0$. In this sense, we adapt the ROW method to nonlinear equations $g(y, z)=0$, using the new parameters $\Delta$ to fulfill (3.11).

\section{AdAPted ROW METHODS}

Let $R$ be defined as in the example of the preceding section. Then (3.11) implies $\alpha=(R-I) \Delta$ and $B=\alpha+\Delta=R \Delta$. Thus $\alpha=\left(I-R^{-1}\right) B$, i.e., $b_{i j}=\alpha_{i+1, j}, i=1(1) s-1, j=1(1) s$. We have to ensure $\alpha_{i+1, i} \neq 0$ for $i=1(1) s-1$ in order to assure regularity of $B$ or $\Delta$, respectively. Then the second condition in (3.11) reads $\mu^{\mathrm{T}} B^{-1} R=e^{\mathrm{T}}$ or $\mu^{\mathrm{T}} B^{-1}=e^{\mathrm{T}} R^{-1}=e_{s}^{\mathrm{T}}$, implying $\mu^{\mathrm{T}}=e_{s}^{\mathrm{T}} B$ or $b_{s j}=\mu_{j}, j=1(1) s$, where $\mu_{s} \neq 0$ is assumed for regularity. Hence, the structure of the adapted ROW-method is exhibited, and the results are collected in the following lemma.

Lemma (Structure of the adapted ROW-method). Let $\mu \in \mathbb{R}^{s}, \mu_{s} \neq 0, \alpha \in$ $\mathbb{R}^{s, s}$ be a lower triangular matrix with vanishing diagonal, and $\alpha_{i+1, i} \neq 0$, 
$i=1(1) s-1$. Define $B$ by

$$
\begin{array}{ll}
b_{i j}:=\alpha_{i+1, j}, & i=1(1) s-1, j=1(1) i, \\
b_{s j}:=\mu_{j}, & j=1(1) s .
\end{array}
$$

Then

(i) $B$ is regular.

(ii) $\alpha B^{-1}=I-R^{-1}$, i.e., $\alpha B^{-1} x=\left(0, x_{1}, \ldots, x_{s-1}\right)^{\mathrm{T}}$, and $\mu^{\mathrm{T}} B^{-1}=e_{s}^{\mathrm{T}}$.

(iii) $\Delta:=B-\alpha=R^{-1} B=B-\left(I-R^{-1}\right) B$.

(iv) (3.11) holds.

(v) $\mu^{\mathrm{T}} B^{-1} e=1$, i.e., the method is stable.

Proof. (i) By assumption. (ii) By (i) and the definition of $B$. (iii) By (ii). (iv) One has $\alpha \Delta^{-1}=\alpha B^{-1} R=R-I$ by (ii), (iii), and $\mu^{\mathrm{T}} B^{-1} R=e_{s}^{\mathrm{T}} R=e^{\mathrm{T}}$ by the definition of $B$ and $R$. (v) By (ii).

The special structure given by (4.1) has some effect on the transformed version of the ROW method and facilitates its implementation:

Adapted ROW method (transformed version):

$$
\begin{gathered}
\left(I-h \gamma_{i i} f_{y}^{0}\right) \kappa_{i}-h \gamma_{i i} f_{z}^{0} \lambda_{i} \\
=\delta_{i i}\left\{h f\left(y_{0}+\sum_{j=1}^{i-1} \kappa_{j}, z_{0}+\sum_{j=1}^{i-1} \lambda_{j}\right)+h f_{y}^{0} \sum_{j=1}^{i-1}\left(\Gamma \Delta^{-1}\right)_{i j} \kappa_{j}\right. \\
\left.+h f_{z}^{0} \sum_{j=1}^{i-1}\left(\Gamma \Delta^{-1}\right)_{i j} \lambda_{j}-\sum_{j=1}^{i-1}\left(\Delta^{-1}\right)_{i j} \kappa_{j}\right\}, \quad i=1(1) s, \\
g_{y}^{0} \kappa_{i}+g_{z}^{0} \lambda_{i}=-g\left(y_{0}+\sum_{j=1}^{i-1} \kappa_{j}, z_{0}+\sum_{j=1}^{i-1} \lambda_{j}\right), \quad i=1(1) s, \\
y_{1}=y_{0}+\sum_{i=1}^{s} \kappa_{i}, \quad z_{1}=z_{0}+\sum_{i=1}^{s} \lambda_{i} .
\end{gathered}
$$

Reminder: $\gamma_{i i} \in\{0, \gamma\}, i=1(1) s$, and $\gamma \neq 0$.

The effect on the order conditions follows from (ii) of the lemma leading to (4.5) Two simplifying rules for the order conditions of an adapted ROW-method:

(1) Replace $\mu^{\mathrm{T}} B^{-1} x=\sigma$ by $x_{s}=\sigma$.

(2) Replace $\alpha B^{-1} x$ by $\left(0, x_{1}, x_{2}, \ldots, x_{s-1}\right)^{\mathrm{T}}$.

Hence, all equations for the $z$-component are considerably simplified. The construction of particular methods thus becomes an easier task. Nevertheless, a further simplification of the order conditions proves to be quite useful. For this reason we introduce the following

(4.6) Simplifying assumption: $A e=\frac{1}{2} B^{-1}(\alpha e)^{2}$. 
This implies $a_{11}=0=\gamma_{11}$ and $\alpha A e=\frac{1}{2} \alpha B^{-1}(\alpha e)^{2}$, i.e., $s-1$ additional equations $(\alpha A e)_{i}=\frac{1}{2}(\alpha e)_{i-1}^{2}, i=2(1) s$. On the other hand, many equations (depending on the desired order, cf. Table 2) are redundant. For example, assumption (4.6) implies (cf. Table 1) that $(2)=(3),(5)=(6),(8)=(9)$, etc.

TABLE 2

Effect of the simplifying assumption (4.6) $N:=$ number of order conditions for an ROW method of order $p$

\begin{tabular}{ccc}
\hline$p$ & $N$ & $(4.6)$ \\
\hline & & \\
2 & 2 & 2 \\
3 & 6 & $4+s$ \\
4 & 18 & $12+s$ \\
5 & 63 & $39+s$ \\
\hline
\end{tabular}

\section{METHODS OF ORDER 2, 3, AND 4}

If $s=1$, there exists an adapted ROW method of order $p=2$ which is uniquely determined by the order equations (1), (2), and by (4.1):

$$
\begin{gathered}
\left(I-h f_{y}^{0} / 2\right) \kappa_{1}-h f_{z}^{0} \lambda_{1} / 2=h f\left(y_{0}, z_{0}\right), \\
g_{y}^{0} \kappa_{1}+g_{z}^{0} \lambda_{1}=-g\left(y_{0}, z_{0}\right) \\
y_{1}=y_{0}+\kappa_{1}, \quad z_{1}=z_{0}+\lambda_{1} .
\end{gathered}
$$

If $s=2$, there exist exactly two adapted ROW methods of order $p=3$ (here and in the sequel we present only the coefficients needed to implement (4.2); $\delta_{i i}=1 /\left(\Delta^{-1}\right)_{i i}$ and $\left.\gamma_{i i}=\delta_{i i}\left(\Gamma \Delta^{-1}\right)_{i i}\right)$ :

$$
\Gamma \Delta^{-1}=\left(\begin{array}{cc}
0 & 0 \\
\sigma / 2 & 1
\end{array}\right), \quad \Delta^{-1}\left(\begin{array}{cc}
\sigma & 0 \\
3-2 \sigma & 3
\end{array}\right), \quad \sigma \in\{-1,1\} .
$$

If $1 / \sigma=\delta_{11}=\alpha_{21}=1$, then the order condition (7) is fulfilled also.

If $s=3$, the situation becomes more interesting, but it is impossible to achieve order $p=4$ with an adapted ROW method. (The same result holds for Roche's ROW method, cf. [14].) Indeed, conditions (3) and (7) imply $\alpha_{31}+\alpha_{32}=1$. Thus (9) forces $\alpha_{21}$ to be in $\{-1,1\}$. But then it is impossible to fulfill (1), (4), (10), and (14) simultaneously.

For ODE's, there exists a 3-stage ROW method of order 4 (see [5]). On the other hand, the method given in $\S 3$ (with $s=3$ ) also has order 4 for the implicit function problem-as does any preconsistent adapted ROW method with $\alpha e=(0, \pm 1,1)^{\mathrm{T}}$. But then it is impossible to achieve order 5 because of condition (28). So we are interested in 3-stage methods with $\alpha e=(0, \pm 1,1)^{\mathrm{T}}$. Then the simplifying assumption (4.6) ensures that even conditions (2) and (8) hold. Furthermore, we are looking for an embedded method of order two, i.e., 
for additional coefficients $\hat{\mu}$ such that conditions (1) and (2) are still satisfied if $\mu$ is replaced by $\hat{\mu}$. Then

$$
\hat{y}_{1}:=y_{0}+\sum_{i=1}^{s}\left(\hat{\mu}^{\mathrm{T}} \Delta^{-1}\right)_{i} \kappa_{i}, \quad \hat{z}_{1}:=z_{0}+\sum_{i=1}^{s}\left(\hat{\mu}^{\mathrm{T}} \Delta^{-1}\right)_{i} \lambda_{i}
$$

can be used for stepsize control. The following are two methods satisfying (4.6):

$$
\begin{gathered}
\hat{\mu}^{\mathrm{T}} \Delta^{-1}=(1,1,5 / 4) \\
\Gamma \Delta^{-1}=\left(\begin{array}{ccc}
0 & & \\
1 / 2 & 0 & \\
1 / 2 & 2 & 9
\end{array}\right), \quad \Delta^{-1}=\left(\begin{array}{lll}
1 & & \\
1 & 3 & \\
1 & 3 & 9
\end{array}\right)
\end{gathered}
$$

and an explicit one:

$$
\Gamma \Delta^{-1}=\left(\begin{array}{ccc}
0 & & \\
1 / 2 & 0 & \\
1 / 2 & 2 & 0
\end{array}\right), \quad \hat{\mu}^{\mathrm{T}} \Delta^{-1} \text { and } \Delta^{-1} \text { as in }(5.4)
$$

In method (5.5), $\gamma_{i i}=0, i=1(1) s$. Hence, (4.2) can be solved explicitly for $\kappa_{1}, \kappa_{2}, \kappa_{3}$. Clearly, a whole class of such methods exists. But we now study the case $s=4$ and show that an adapted ROW method of order $p=4$ does exist, in contrast to Roche's approach. Furthermore, a method of order 3 can be embedded. The order $p=5$ is already impossible to achieve for ODE's (cf. [5]). Moreover, an adapted 4-stage ROW method of order four cannot have order five for the implicit function problem. Indeed, the conditions (3), (7), (9) imply $(\alpha e)_{4}=1$ and $(\alpha e)_{3}^{2}=1$. Then (19), (24), and (29) are fulfilled. The remaining conditions are $(27),(28)$, i.e., $(\alpha e)_{3}=1$ and $(\alpha e)_{2}^{2}=1$. If they hold, then the linear equations for $\mu$ arising from (1), (4), (10), (14) would have no solution. Hence, if $(\alpha e)_{3}=1$, then we necessarily need $(\alpha e)_{2}^{2} \neq 1$.

Furthermore, the following results hold:

(1) If $(\alpha e)_{3}=1$ and $\alpha_{21}^{2} \neq 1$, then $\alpha_{21}$ must not be equal to $\tau \approx-0.928$, where $\tau$ is the only real root of the polynomial $4 t^{3}-3 t^{2}-3 t+3$. Otherwise, $\mu_{4}$ would vanish, contrary to the regularity of $\Delta$.

(2) If $(\alpha e)_{3}=-1$, then $\tau_{1}, \tau_{2}$ are forbidden values for $\alpha_{21}$. Here, $\tau_{1}$ denotes the real zero of $t^{3}-t^{2}+t+1$ which leads to unsolvability for $\mu$, and $\tau_{2}$ denotes the real zero of $4 t^{3}-3 t^{2}+3 t+3$, leading to $\mu_{4}=0$.

(3) In all other cases, an adapted ROW method of order 4 (with four stages) can be constructed; but it is never explicit, i.e., $\operatorname{diag}(\Gamma) \neq 0$.

These results are due to $\mathrm{W}$. Link (University of Mainz, private communication). We restrict ourselves to the case $(\alpha e)_{3}=-1, \alpha_{21}=1$, and assume that (4.6) holds. This yields - after lengthy calculations-the following class of 
4-stage adapted ROW methods of order 4:

$$
\begin{aligned}
\alpha_{32} \neq & 0, \quad \alpha_{43} \neq 0, \quad a_{22}, a_{33} \in\{0,7 / 24\} \\
\alpha_{21}= & 1, \quad \alpha_{41}=4 / 3, \quad a_{11}=0, \quad a_{44}=7 / 24, \\
\mu^{\mathrm{T}}= & (16,0,1,7) / 24, \\
\alpha_{31}= & -1-\alpha_{32}, \quad \alpha_{42}=-1 / 3-\alpha_{43}, \\
a_{21}= & 1 /\left(2 \alpha_{32}\right)-a_{22}, \quad a_{43}=1 / 24+\alpha_{43} / 7-a_{33} / 7, \\
a_{42}= & -\alpha_{43} / 7-\left(1 / 3-a_{33}+a_{22}\right) / 7 \\
& -\left(a_{22}-a_{33}-\alpha_{32}-3 \alpha_{32} a_{33}\right) /\left(21 \alpha_{43}\right), \\
a_{32}= & -1 / 3-\alpha_{43}-7 a_{42}, \\
a_{41}= & -1 /\left(42 \alpha_{32} \alpha_{43}\right)-1 /\left(14 \alpha_{43}\right)-1 /\left(14 \alpha_{32}\right)+10 / 7 \\
& +a_{22} / 7+\left(a_{22}-a_{33}-\alpha_{32}-3 \alpha_{32} a_{33}\right) /\left(21 \alpha_{43}\right), \\
a_{31}= & 10-7 a_{41} .
\end{aligned}
$$

We now look for an embedded method of order three, i.e., for $\hat{\mu}$ such that conditions (1)-(6) still hold but at least one of the conditions (7)-(18) does not. With the assistance of W. Link, and using MACSYMA, we find the condition $a_{22}=\alpha_{32}$, leading to

$$
\begin{aligned}
& \alpha_{32}=a_{22}=7 / 24, \quad \hat{\mu}_{1}=2 / 3, \\
& \hat{\mu}_{4} \neq 7 / 24, \\
& \hat{\mu}_{3}=\alpha_{43}\left(24 \hat{\mu}_{4} / 7-1\right) / 15+\hat{\mu}_{4} / 7, \\
& \hat{\mu}_{2}=1 / 3-\hat{\mu}_{3}-\hat{\mu}_{4} .
\end{aligned}
$$

The remaining free parameters can be used to minimize the error constant. This yields the following

(5.8) Embedded adapted 4-stage ROW method of order 4(3):

$$
\Gamma \Delta^{-1}=\frac{1}{7}\left[\begin{array}{cccc}
0 & & \\
19 & 7 & \\
53 / 2 & 0 & 0 & \\
3 / 14 & 0 & 1 & 7
\end{array}\right], \quad \Delta^{-1}=\frac{1}{7}\left[\begin{array}{rrrr}
7 & & \\
55 & 24 & & \\
71 & 39 & 7 & \\
-15 / 7 & 129 / 7 & 23 & 24
\end{array}\right] .
$$

If $s=5$, it is impossible to achieve order $p=5$ with an adapted ROW method, as can be seen by inspecting the conditions for $\alpha e$, leading to $(\alpha e)^{\mathrm{T}}=$ $\left(0, \alpha_{21},-1,1,1\right)$, which is not compatible with the conditions connecting $\mu$ and $\alpha e$. But there exists a whole family of adapted ROW methods with $s=5$ and $p=4$ which fulfill the order conditions (1)-(30), i.e., they converge with order $p=5$ for the implicit function problem, and with order 6 for purely 
nonlinear equations. An explicit one is given by the following data:

(5.9) Embedded explicit 5-stage adapted ROW method of order 4(3):

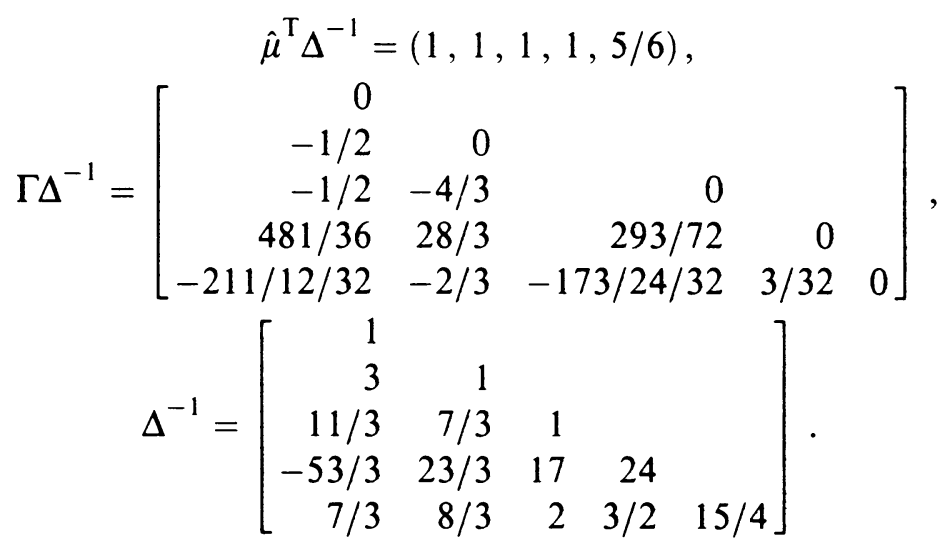

The methods described in this section have been implemented and applied to general DAE's and to special DAE's like implicitly given ODE's or implicitly defined functions as well. Compared with the methods given in [12] and [14], the adapted ROW methods yield better results in the $z$-component. For example, method (5.8) solves the numerical problem described in [14] better than Roche's 5-stage method: the $y$-component is better approximated by a factor 4 , the $z$-component by a factor 100 . Hence, we remain closer to the manifold defined by the function $g$. This has been the purpose of our approach-to embed a fast solver for nonlinear equations in the ROW method.

\section{BIBLIOGRAPHY}

1. P. Deuflhard, E. Hairer, and J. Zugck, One-step and extrapolation methods for differentialalgebraic systems, Numer. Math. 51 (1987), 501-516.

2. C. W. Gear and L. Petzold, $O D E$ methods for the solution of differential algebraic systems, SIAM J. Numer. Anal. 21 (1985), 716-728.

3. E. Griepentrog and R. März, Differential-algebraic equations and their numerical treatment, Teubner Text zur Mathematik, no. 88, Teubner, Leipzig, 1986.

4. E. Hairer, C. Lubich, and M. Roche, The numerical solution of differential-algebraic systems by Runge-Kutta methods, Lecture Notes in Math., vol. 1409, Springer, New York, 1989.

5. P. Kaps and G. Wanner, A study of Rosenbrock-type methods of high order, Numer. Math. 38 (1981), 279-298.

6. W. Liniger, Multistep and one-leg methods for implicit mixed differential algebraic systems, IEEE Trans. Circuits and Systems CAS-29 (1979), 755-762.

7. P. Lötstedt and L. Petzold, Numerical solution of nonlinear differential equations with algebraic constraints. I: Convergence results for backward differentiation formulas, Math. Comp. 46 (1986), 491-516.

8. R. März, Multistep methods for initial value problems in implicit differential-algebraic equations, Beiträge Numer. Math. 12 (1984), 107-123.

9. S. P. Nørsett and A. Wolfbrandt, Order conditions for Rosenbrock type methods, Numer. Math. 32 (1979), 1-15.

10. L. Petzold, Differential-algebraic equations are not ODE's, SIAM J. Sci. Statist. Comput. 3 (1982), 367-384. 
11. L. Petzold and P. Lötstedt, Numerical solution of nonlinear differential equations with algebraic constraints. II: Practical applications, SIAM J. Sci. Statist. Comput. 7 (1986), 720-731.

12. P. Rentrop, M. Roche, and G. Steinebach, The application of Rosenbrock-Wanner type methods with stepsize control in differential-algebraic equations, Numer. Math. 55 (1989), 545-563. ${ }^{1}$

13. W. C. Rheinboldt, Differential-algebraic systems as differential equations on manifolds, Math. Comp. 43 (1984), 473-482.

14. M. Roche, Rosenbrock methods for differential-algebraic equations, Numer. Math. 52 (1988), 45-63.

15. H. H. Rosenbrock, Some general implicit processes for the numerical solution of differential equations, Comput. J. 5 (1963), 329-330.

16. A. Wolfbrandt, $A$ study of Rosenbrock processes with respect to order conditions and stiff stability, Thesis, Chalmers University of Technology, Göteborg, Sweden, 1977.

17. J. F. Traub, Iterative methods for the solution of equations, Prentice-Hall, Englewood Cliffs, N. J., 1964.

Johannes Gutenberg-Universität, Fachbereich Mathematik, SaArstrasse 21, D-6500 Mainz, West Germany

E-mail address: csch@dmzrzu71.bitnet

\footnotetext{
${ }^{1}$ We call attention to four wrong coefficients in this paper: The method DAE34 has order $p=4$, if (cf. Table (3.7)), $\wedge_{41}=-0.005208 \overline{3} \ldots, \kappa_{42}=0.192708 \overline{3} \ldots, \bar{\gamma}_{41}=1.1041 \overline{6} \ldots, \bar{\gamma}_{42}=$ $-0.94791 \overline{6} \ldots$.
} 